\title{
Applying Ontology-Informed Lattice Reduction Using the Discrimination Power Index to Financial Domain
}

DOI:

10.1007/978-3-030-19037-8_11

\section{Document Version}

Accepted author manuscript

Link to publication record in Manchester Research Explorer

\section{Citation for published version (APA):}

Quboa, Q., Mehandjiev, N., \& Behnaz, A. (2019). Applying Ontology-Informed Lattice Reduction Using the Discrimination Power Index to Financial Domain. In N. Mehandjiev, \& B. Saadouni (Eds.), Enterprise Applications, Markets and Services in the Finance Industry (Vol. 345, pp. 165-179). Springer Nature.

https://doi.org/10.1007/978-3-030-19037-8_11

Published in:

Enterprise Applications, Markets and Services in the Finance Industry

\section{Citing this paper}

Please note that where the full-text provided on Manchester Research Explorer is the Author Accepted Manuscript or Proof version this may differ from the final Published version. If citing, it is advised that you check and use the publisher's definitive version.

\section{General rights}

Copyright and moral rights for the publications made accessible in the Research Explorer are retained by the authors and/or other copyright owners and it is a condition of accessing publications that users recognise and abide by the legal requirements associated with these rights.

\section{Takedown policy}

If you believe that this document breaches copyright please refer to the University of Manchester's Takedown Procedures [http://man.ac.uk/04Y6Bo] or contact uml.scholarlycommunications@manchester.ac.uk providing relevant details, so we can investigate your claim.

\section{OPEN ACCESS}




\title{
Applying Ontology-informed Lattice Reduction Using the Discrimination Power Index to Financial Domain
}

\author{
Qudamah Quboa ${ }^{1}$, Nikolay Mehandjiev² and Ali Behnaz ${ }^{3}$ \\ ${ }^{1}$ Alliance Manchester Business School, University of Manchester, UK \\ ${ }^{2}$ School of Computer Science and Engineering, University of New South Wales, Australia \\ \{qudamah.quboa, n.mehandjiev\}@manchester.ac.uk, \\ ali.behnaz@unsw.edu.au
}

\begin{abstract}
Contemporary financial institutions are relying on varied and voluminous data and so they need advanced technologies to provide their customers with the best possible services. Capturing the meaning, or semantics, of data and presenting these semantics in simplified yet relevant models are key challenges to achieving this. Formal Concept Analysis (FCA) automates the analysis of properties and instances of the data, generating a lattice which groups properties and instances into concepts. This lattice can be used as automatically generated semantic structure describing the domain, yet the complexity and size of the resultant lattice render this technique unusable in most practical cases involving financial data. To tackle this, our Ontology-informed Lattice Reduction approach can guide the reduction of the lattices generated from financial sampled data. We validate the adaptation of the approach to the financial domain through a real-world asset allocation case study, demonstrating that the approach achieves good overall performance and relevant results.
\end{abstract}

Keywords: FCA, Semantic structures, Lattice reduction, Validation.

\section{Introduction}

The financial industry has been undergoing immense changes and disruption in the last decade. Indeed, one can argue that no force has influenced the industry more than abundant data and cheap computational power. Modern financial institutions are focused on excellence through advanced information technologies. To provide stateof-the-art customer service, financial institutions collect vast amount of data including news data, financial market data, sentiment data and data from other exogenous factors. However, the heterogeneous nature of this data makes it difficult for players in financial industry to obtain real benefits from their data. One key obstacle in leveraging data assets is difficulties in capturing the meaning (semantics) of the data. Therefore, knowledge acquisition is a key step in the process and its automation will be an enabler. Automating knowledge acquisition involves [1], processing voluminous data [2], understanding the meaning of this data and its relations [3] and presenting the outcomes in relevant yet simplified models [4]. 
For practical use, manual semantic tagging of data is very expensive task and many scientific researchers are working to automate this task. As an example, most data in financial markets has high volume and needs to be processed at a high speed to provide value for its users. Formal Concept Analysis (FCA) is one technique for tagging semantics to data. FCA takes a matrix of incidence relationships between sampled data properties (intent) and their object instances (extent), named a formal context, and then builds a lattice of partial order relations between the two sets (instance and property sets). One major issue here is the complexity and nosiness of lattices produced by FCA to be used for practical semantic analysis of real-world datasets. To solve this issue, a reduction of lattices is required and existing approaches to achieve that are based on mathematical measurements of relevancy [4]. They are agnostic about any prior knowledge regarding the targeted domain, even when it is already formalised and represented in an ontology or semantic structure.

Inspired by the similarities between ontology-based semantic and FCA representations, different approaches have been proposed to use the combination of both such as ontology modelling and attribute exploration $[5,6]$ and merging different ontologies[7,8]. However, the use of existing domain knowledge (represented as ontology) to support the lattice reduction has not been explored until now. In our research [9], we proposed Ontology-informed Lattice Reduction approach that address the attaching of semantics to further instances in the domain through FCA using existing domain ontology.

The approach uses prior domain knowledge (encoded in semantic format ontology) to classify and guide the reduction process of a sampled formal context where not all instances are in the ontology. In addition, the approach relies on a new relevancy metric called Discrimination Power Index (DPI) that is used to automatically classify any new instances based on the shared instances and the overall power of a property within the formal context.

In this paper, we present a more detailed analysis and testing of the proposed approach [9] to evaluate and validate the performance of the approach especially when facing the problem of large lattices generated when applying FCA to real-world data. A financial real-world case study is presented to confirm the feasibility and the validity of the approach.

The remainder of this paper is structured as follows: Section 2 describes shortly the background of the work. Sections 3 summarises briefly the proposed approach and its stages. Section 4 introduces a real-world case study in financial markets to evaluate the adaption of the approach. Then Section 5 discusses the results and the statistical analysis of applying the approach and Section 6 concludes the paper.

\section{$2 \quad$ Literature Review}

\subsection{Analytics in Financial Domain}

As data is becoming more abundant, organizations are looking for ways to acquire actionable insights from their data, and hence make better decisions, achieve value and competitiveness. Domain experts and analytics units at organizations have access to sophisticated analytics solutions which serve their users' ways of conducting analytics. 
For instance, a bank would be interested to forecast price moves in a particular asset. There are thousands of assets (instances) to be analysed, and for each asset hundreds of properties (intents) can be input into a predictive model to forecast the changes in the respective asset $[10,11]$.

A typical analytics problem in finance domain has heterogeneous complicated datasets which need to be understood easily and acted upon. As a result, knowledge acquisition and knowledge representation are key elements in enhancing big data analytics problems. Semantic web technology and ontologies present a solution to capture knowledge in a domain [12].

\subsection{Semantic Web Ontology}

Ontology is a well-known knowledge representation method and widely supported by both the academic and the industry domains in terms of available software and tools. Ontologies defines as "explicit formal specifications of the terms in the domain and relations among them" [12]. Different general and specialist ontologies in various domains have developed by experts to capture knowledge and pass information in standardised way.

It is mainly designed to define a set of data and its structure, constrains for the use of other applications, and commonly utilised as a data sharing mechanism between various programs or software agents. For instance, FIBO (the Financial Industry Business Ontology) is an "industry initiative to define financial industry terms, definitions and synonyms using semantic web principles [13]."

\subsection{Formal Concept Analysis (FCA)}

Formal Concept Analysis (FCA) is a mathematical formalism to automatically analyse the structure of a domain of interest [2]. It generates a lattice of concepts representing incidence relations between sets of observed properties (intent) and their object instances (extent) in a target domain. The generated lattice is constituted by formal concepts produced from mapping these relationships onto a knowledge structure that reflects the specialisation and generalisation among the concepts of resultant lattice [2].

"Sports and their attributes" [14] (presented in Table 1) is an example of formal context $(\mathrm{K})$. In this example, the extent set $(\mathrm{G})$ is \{Run, Gymnastics, Triathlon, Football, Tennis, Baseball, Curling, Diving, Rowing\}, the intent set (M) is \{on land, on ice, in water, collective sport, individual sport, using ball, needs opponent, multiple disciplines $\}$ and the sign $\mathrm{X}$ identifies a relation between instances of $\mathrm{G}$ and properties of $\mathrm{M}$ (representing the set of I). 
Table 1. "Sports and their attributes" formal context (taken from [14]).

\begin{tabular}{|c|c|c|c|c|c|c|c|c|}
\hline & on land & on ice & in water & $\begin{array}{c}\text { collective } \\
\text { sport }\end{array}$ & $\begin{array}{c}\text { individual } \\
\text { sport }\end{array}$ & using ball & $\begin{array}{c}\text { needs } \\
\text { opponent }\end{array}$ & $\begin{array}{c}\text { multiple } \\
\text { disciplines }\end{array}$ \\
\hline Run & $\mathrm{X}$ & & & & $\mathrm{X}$ & & & \\
\hline Gymnastics & $\mathrm{X}$ & & & & $\mathrm{X}$ & & & $\mathrm{X}$ \\
\hline Triathlon & $\mathrm{X}$ & & $\mathrm{X}$ & & $\mathrm{X}$ & & & $\mathrm{X}$ \\
\hline Football & $\mathrm{X}$ & & & $\mathrm{X}$ & & $\mathrm{X}$ & $\mathrm{X}$ & \\
\hline Tennis & $\mathrm{X}$ & & & $\mathrm{X}$ & & $\mathrm{X}$ & $\mathrm{X}$ & \\
\hline Baseball & $\mathrm{X}$ & & & $\mathrm{X}$ & & $\mathrm{X}$ & $\mathrm{X}$ & \\
\hline Curling & & $\mathrm{X}$ & & $\mathrm{X}$ & & & & \\
\hline Diving & & & $\mathrm{X}$ & & $\mathrm{X}$ & & & \\
\hline Rowing & & & $\mathrm{X}$ & $\mathrm{X}$ & & & & \\
\hline
\end{tabular}

By processing the relationships between properties and instances of this example, the corresponding concept lattice will be formed, as shown in Fig. 1, where each node represents a formal concept and each connecting edge represents a subconceptsuperconcept relationship.

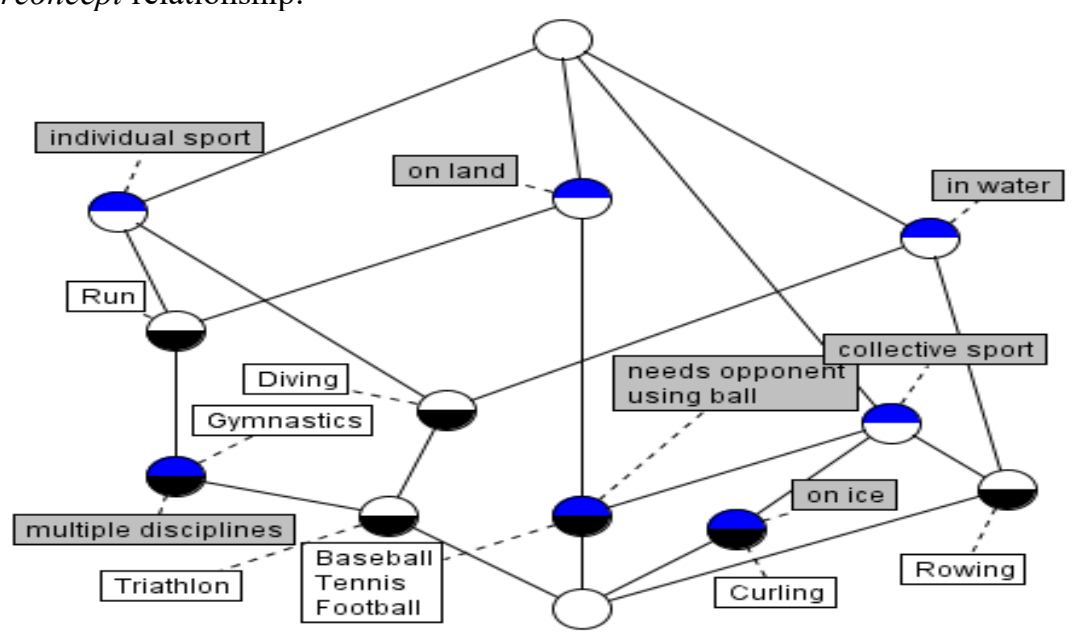

Fig. 1. Concept Explorer-generated FCA lattice of "Sports and their attributes" example [14].

\subsection{Existing Lattice Reduction Techniques}

A bottleneck problem with FCA mechanism is the huge size of lattices created from real-world data sets because of noise and exceptions [2, 15]. Various methods have been proposed to reduce the lattice using the structure of the lattice itself. These are categorised into redundant information removal, simplification or selection [4].

The focus of the work is the last category, the selection reduction, which represents any approach that emphases on selecting specific properties, instances and/or concepts based on different measurements of relevancy (a set of constraints that 
requires to be satisfied). This could be depended on various measurements, such as logic (according to a user's attributes priorities [16]), weight (frequent weighted concept reduction [17]), or hierarchies (using hierarchically ordered attributes [18]). This kind of reduction is performed after completing the construction of the formal context [4].

All these approaches mainly rely on the lattice structure and are agnostic about any prior knowledge about the domain that makes the results more vulnerable to systemic noise in the data.

\subsection{Similarity Measurements}

Three similarity measurements are used to align the two different formal contexts (ontology-derived and sampled) and integrate them. The first two are:

Jaccard Similarity Coefficient Index. This well-known similarity measurement [19] is based on the following formula:

$$
\text { Jaccard Index } S_{J a c}=\frac{\left|B_{1} \cap B_{2}\right|}{\left|B_{1} \cup B_{2}\right|}
$$

Hamming Distance Index. This is also a well-known similarity measurement [20] and is based on the following formula:

$$
D_{\text {hamming }}=b+c
$$

It is worth to mention that these two measurements weight all properties as of equal importance whilst our observations reveal that specific properties could have a higher discrimination power than others.

We thus introduced a new complementary index called Discrimination Power Index (DPI) [9] to identify a unique most-similar concept. It enhances the similarity selection process in picking one of the possible concepts (pre-filtered from the previous indices) based on their properties' overall discrimination power. This is formally defined as [9]:

$$
D P I=\frac{\left|\left\{\forall g \in G \mid b \in B_{1} \cap B_{2}:(g, b) \in I\right\}\right|}{|G|}
$$

The theoretical explanations of each of the mentioned equations are covered in [9].

\section{Ontology-informed Lattice Reduction Approach}

Our approach presented in [9] uses existing knowledge about the target domain (encoded in a semantic ontology format) to support reduce the FCA-generated lattice when extracting structure from sampled formal context (data). The approach begins by extracting and transforming all required information into acceptable formats. It then starts by recognising object instances that exist in the formal context and the ontologyderived context and aligns concepts using these identified instances. This is followed by automatically structures the rest of object instances from the formal context, using (a) basic alignment routine that rely on the properties they have in common with the 
shared instances and (b) advanced alignment routine that is based on the similarity measurements and the discrimination power of the properties. At the end, the resultant extended structure will be reduced based on the user's reduction threshold. The general outline of the approach shown in Fig. 2.

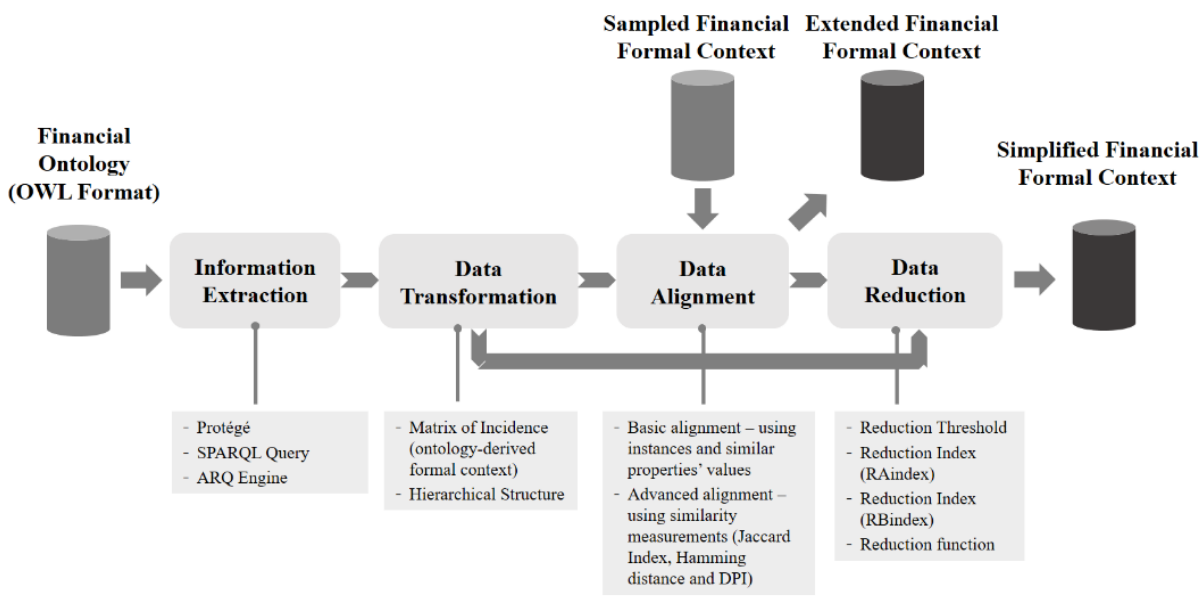

Fig. 2. The outline of the Ontology-informed Lattice Reduction approach [adapted from 9].

In the next subsections, a summary of each stage of the proposed approach (presented in Fig. 2) is provided.

\subsection{Data Extraction:}

During this stage, Protégé, a well-known Ontology editor and knowledge acquisition software, is used to establish a deep understanding of the domain ontology as well as helping in the construction of the constrains of the retrieval queries.

To retrieve the required features and their instances from any Semantic Web dataset, it is necessary to use a semantic query language. SPARQL, recommended by W3C, is used as a simple protocol and Semantic Web query language to perform the querying via pattern matching [21].

Lastly, ARQ engine is a query engine for Jena (Java framework for building Semantic Web applications), which provides the support to the standard SPARQL query language, is used to execute the SPARQL queries, extract the results, and save them into temporary comma-separated values (csv) file that will be used in the next stage.

\subsection{Data Transformation:}

The main purpose of the stage is to reformat the extracted information from the ontology source into a formal context format (in a tabulated format) and support it with transforming the hierarchal structure of the extracted data based on the ontology source itself and storing the results in a matrix format to easy the access. 
The developed algorithms for this stage support multiple hierarchal levels of ontology structure and for any number of instances. This stage works as a prepossessing stage to complete all the required preparations to align the different datasets and reducing the results later on.

\subsection{Data Alignment}

Initially, a basic matching routine is applied based on instances existing in both the ontology and the sampled formal context. This is followed by a more complex classification routine for any instance not existing in the ontology and none of the classified instances shares the same properties. This is achieved by assigning any unknown concept from the sampled formal context to one of the existing ontology concepts using a combination of different similarity indices to evaluate and align concepts from both contexts based on their intents and extents.

This advanced alignment routine starts with a Jaccard similarity coefficient index followed by optional use of a Hamming distance, and then the proposed DPI [9] (again optional). The outcome of this stage is an extended formal context incorporating both the ontology-derived knowledge and the sampled formal context.

\subsection{Data Reduction}

Two indices are proposed in [9] to provide an indication whether a property is essential to a specific concept from the ontology or not:

RAindex is the first reduction index that focuses on evaluating the weight of every property in the sampled formal context regarding each and every extracted concepts from the domain ontology.

RBindex is a complementary index to indicate the concepts that need the reduction by relying on both the property and the ontology's concept in making the call.

Depending on the outcomes of both RAindex and RBindex indices, the reduction function is executed when required. The purpose of this function is to eliminate the incidence relations between the assessed property related to the used ontology concept and the object instances.

The theoretical explanations and the justifications of each of the mentioned indices and the reduction function are covered in [9]. This stage depends mainly on the sampled formal context and the extracted ontology information and hierarchical structure (prepared in the data extraction and data transformation stages) to work out which of the incidence relationships need to be removed from the extended formal context (resulted from the data alignment stage).

\section{$4 \quad$ Financial Assets Allocation Case Study (Ontology and Formal Context of Exchange Traded Funds)}

In this case study, we use semantic technology to capture and represent the knowledge related to asset allocation. Asset allocation intends to distribute investment among different financial assets so as to achieve a certain investment strategy [22]. The 
knowledge is extracted and represented in an ontology including a list of Exchange Traded Funds $\left(\mathrm{ETF}^{1}\right)$. The goal in this case is to design and build an automated financial advice system which helps people invest and manage their funds at a fraction of the cost for human financial advice. As part of asset allocation, we explore, analyse and select a number of assets (in this case ETFs). We have obtained the data for these ETFs from Bloomberg. In doing so, we have adopted Bloomberg terminology for assets, their properties and respective categories. The sample consists of 87 ETFs (instances) and 42 (properties) presented in the Asset Selection ontology (see Fig. 3). Table 2 provides simple statistics for both the ontology and sampled formal context then Table 3 presents the data description of the financial sampled formal context properties.

Table 2. Basic statistical analysis of the financial sampled formal context and its ontology.

\begin{tabular}{|c|c|c|c|c|c|}
\hline & $\begin{array}{c}\text { No of } \\
\text { Instances }\end{array}$ & $\begin{array}{c}\text { No of Properties } \\
\text { (Classes) }\end{array}$ & $\begin{array}{c}\text { No of } \\
\text { Concepts }\end{array}$ & $\begin{array}{c}\text { No of Levels / } \\
\text { Height }\end{array}$ & $\begin{array}{c}\text { No of } \\
\text { Edges }\end{array}$ \\
\hline $\begin{array}{c}\text { Sampled } \\
\text { Formal Context }\end{array}$ & 87 & 36 & 546 & 9 & 1722 \\
\hline Ontology & 87 & 42 & 44 & 4 & 74 \\
\hline
\end{tabular}

Table 3. The data description of the sampled financial formal context.

\begin{tabular}{|c|c|}
\hline Properties & Possible Values \\
\hline Closed for new creations & $\{$ Yes, No $\}$ \\
\hline Leverage / Short & $\{$ Yes, No\} \\
\hline Invests in Derivatives & $\{$ Yes, No $\}$ \\
\hline Invests in Swaps & $\{$ Yes, No $\}$ \\
\hline $\begin{array}{c}\text { Invests in Physical } \\
\text { Commodities }\end{array}$ & $\{$ Yes, No $\}$ \\
\hline Actively Managed & $\{$ Yes, No\} \\
\hline Currency Hedged & $\{$ Yes, No $\}$ \\
\hline Index Replication Strategy & $\{$ Full, Optimized, Not Applicable, Derivative $\}$ \\
\hline Index Weighting & $\{$ Market Cap, Not Applicable, Single Asset, Multi Factor, \\
Methodology & Fundamentals, Dividend, Proprietary, Equal $\}$ \\
\hline Rebalancing Frequency & $\{$ Quarterly, Not Applicable, Yearly, Semi-Annually, \\
Other, Monthly $\}$ \\
\hline Creation / Redemption & $\{$ In-kind, In-kind/Cash, Not Applicable, Cash $\}$ \\
\hline Dividend Frequency & $\{$ Quarter, Semi-Anl, Annual, None, Monthly, Irreg $\}$ \\
\hline Risk & $\{$ Low, High $\}$ \\
\hline
\end{tabular}

\footnotetext{
${ }^{1}$ Exchange Traded Funds or ETFs are a basket of other assets that are designed to trace the performance of an index
} 


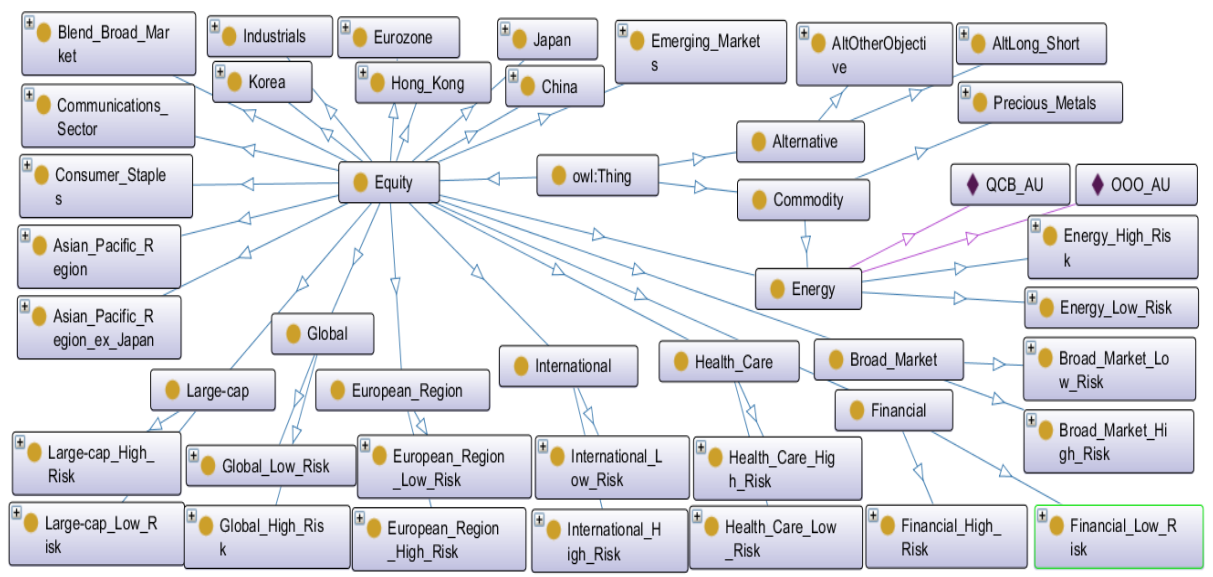

Fig. 3. Asset Selection Ontology.

\section{$5 \quad$ Analysis and Discussion}

To validate the validity of applying the approach in this financial context, different validation measurements are carried out to ensure each and every stage is generating the expected outcome with the right results.

By relying on Protégé, the construction of the SPARQL query is achieved and the retrieved results (using ARQ Engine) have all the main classes and sub-classes and their hierarchal structure levels within the domain ontology. The extracted information is then compared with the ontology itself to confirm the correctness of outcome. This includes matching the number of extracted instances (objects) and their classessubclasses properties.

Moving to the second phase, Data Transformation, the validation here is achieved by comparing the outcome of the previous stage with the transformed results and making sure that all the data are transformed and correctly aligned.

During the Data alignment stage, two measurements are used to evaluate and validate the process: (1) testing the functionality of the basic alignment routine alone, and (2) testing the efficiency of alignment process with the advanced alignment routine (the Jaccard index, Hamming Distance, and DPI).

For the first test, the experiment starts by using only known instances that exist in both the financial sampled formal context and the financial ontology. Then the validation of the alignment routine is confirmed by comparing the outcome of this stage (Extended financial formal context) with the original sources and ensuring that all the instances are classified accurately as they should be.

For the second evaluation, $50 \%$ of the ontology instances are removed to create unknown instances in the sampled financial formal context while the other $50 \%$ are kept to construct the training part for the advanced alignment routine to work. Then the resulted classification outcome is evaluated and compared with the original one to confirm the performance of the alignment. Table 4 presents a summary of the results. It is worth to mention that the accuracy of the alignment for the top level of the ontology 
is $100 \%$ and the reason of having a less accurate rate for the sublevels concepts is the large possible combinations of the properties that do not exist in the training dataset.

Table 4. Alignment Validation Results (50\% Training - 50\% Testing).

\begin{tabular}{|c|c|c|}
\hline $\begin{array}{c}\text { Semantics Attachment Alignment } \\
\text { Accuracy }\end{array}$ & $\begin{array}{c}\text { No of } \\
\text { Instances }\end{array}$ & $\begin{array}{c}\text { Overall } \\
\text { Percentage }\end{array}$ \\
\hline $100 \%$ & 54 & $62 \%$ \\
\hline $95 \%$ & 1 & $1 \%$ \\
\hline $93 \%$ & 10 & $11 \%$ \\
\hline $90 \%$ & 22 & $25 \%$ \\
\hline Below $90 \%$ & 0 & $0 \%$ \\
\hline
\end{tabular}

During the last stage of the applying the approach, the performance of the reduction function is evaluated using two different extended formal contexts: (a) the extended one using the basic alignment routine and (b) the extended one using both alignments routines (50\% Training - 50\% Testing). The performance of the function is evaluated at various reduction thresholds (presented in Table 5 and Table 6 respectively).

Table 5. The reduction performance using the fully classified formal context (the extended formal context) based on the basic alignment routine only.

\begin{tabular}{|c|c|c|c|c|c|c|c|}
\hline 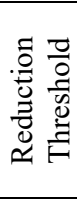 & 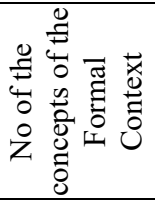 & 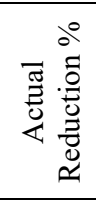 & 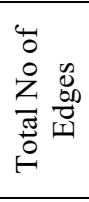 & 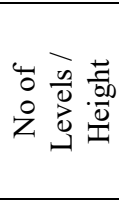 & 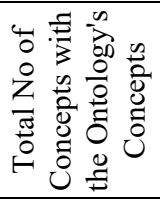 & 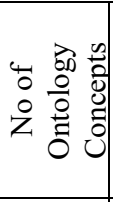 & 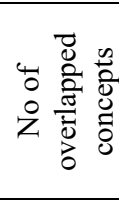 \\
\hline $90 \%$ & 24 & $95.6 \%$ & 40 & 5 & 59 & 44 & 9 \\
\hline $80 \%$ & 24 & $95.6 \%$ & 40 & 5 & 59 & 44 & 9 \\
\hline $70 \%$ & 24 & $95.6 \%$ & 40 & 4 & 61 & 44 & 7 \\
\hline $60 \%$ & 31 & $94.3 \%$ & 54 & 5 & 85 & 44 & 10 \\
\hline $50 \%$ & 97 & $82.2 \%$ & 248 & 8 & 203 & 44 & 62 \\
\hline $40 \%$ & 148 & $72.9 \%$ & 414 & 8 & 293 & 44 & 101 \\
\hline $30 \%$ & 269 & $50.7 \%$ & 849 & 9 & 487 & 44 & 174 \\
\hline $20 \%$ & 324 & $40.7 \%$ & 1030 & 9 & 598 & 44 & 230 \\
\hline $10 \%$ & 477 & $12.6 \%$ & 1519 & 9 & 801 & 44 & 280 \\
\hline $0 \%$ & 546 & $0.0 \%$ & 1722 & 9 & 878 & 44 & 288 \\
\hline
\end{tabular}


Table 6. The reduction performances using the extended formal context based on the basic and advanced alignments routines (50\% Training - 50\% Testing).

\begin{tabular}{|c|c|c|c|c|c|c|c|}
\hline 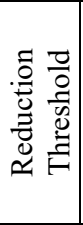 & 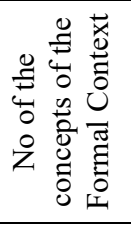 & 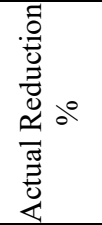 & 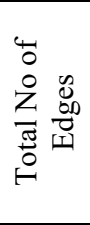 & 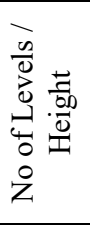 & 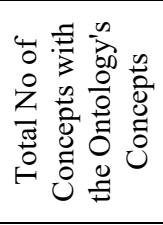 & 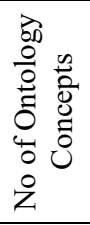 & 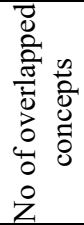 \\
\hline $90 \%$ & 24 & $95.6 \%$ & 39 & 4 & 57 & 44 & 11 \\
\hline $80 \%$ & 24 & $95.6 \%$ & 39 & 4 & 59 & 44 & 9 \\
\hline $70 \%$ & 30 & $94.5 \%$ & 48 & 5 & 69 & 44 & 5 \\
\hline $60 \%$ & 43 & $92.1 \%$ & 76 & 6 & 92 & 44 & 5 \\
\hline $50 \%$ & 121 & $77.8 \%$ & 315 & 8 & 214 & 44 & 49 \\
\hline $40 \%$ & 185 & $66.1 \%$ & 533 & 8 & 303 & 44 & 74 \\
\hline $30 \%$ & 313 & $42.7 \%$ & 984 & 9 & 477 & 44 & 120 \\
\hline $20 \%$ & 334 & $38.8 \%$ & 1058 & 9 & 520 & 44 & 142 \\
\hline $10 \%$ & 486 & $11.0 \%$ & 1546 & 9 & 682 & 44 & 152 \\
\hline $0 \%$ & 546 & $0.0 \%$ & 1722 & 9 & 790 & 44 & 200 \\
\hline
\end{tabular}

Figs. 4 and 5 illustrate the generated lattice of the reduced sampled formal context at a reduction threshold of $60 \%$ for both alignments experiments respectively without the semantic attachments.

It could be noticed that the performance of the approach using the advanced alignment routine (training and testing datasets) is very similar to the actual known one, which reconfirms the efficiency of the alignment stage.

In addition, the reduction function is working as expected and performing well in reducing the sampled data and presenting the results using simplified (and relevant) financial lattice, even when the knowledge base only provides partial coverage of the domain of interest.

It is worth to mention that (1) the reduction threshold represents the minimum ratio to pass the RBindex and not being flagged as an unnecessary concept. (2) The actual reduction is different from the reduction threshold and its value is vary depending on the incidence relationships of the actual formal context and its semantic extension. 


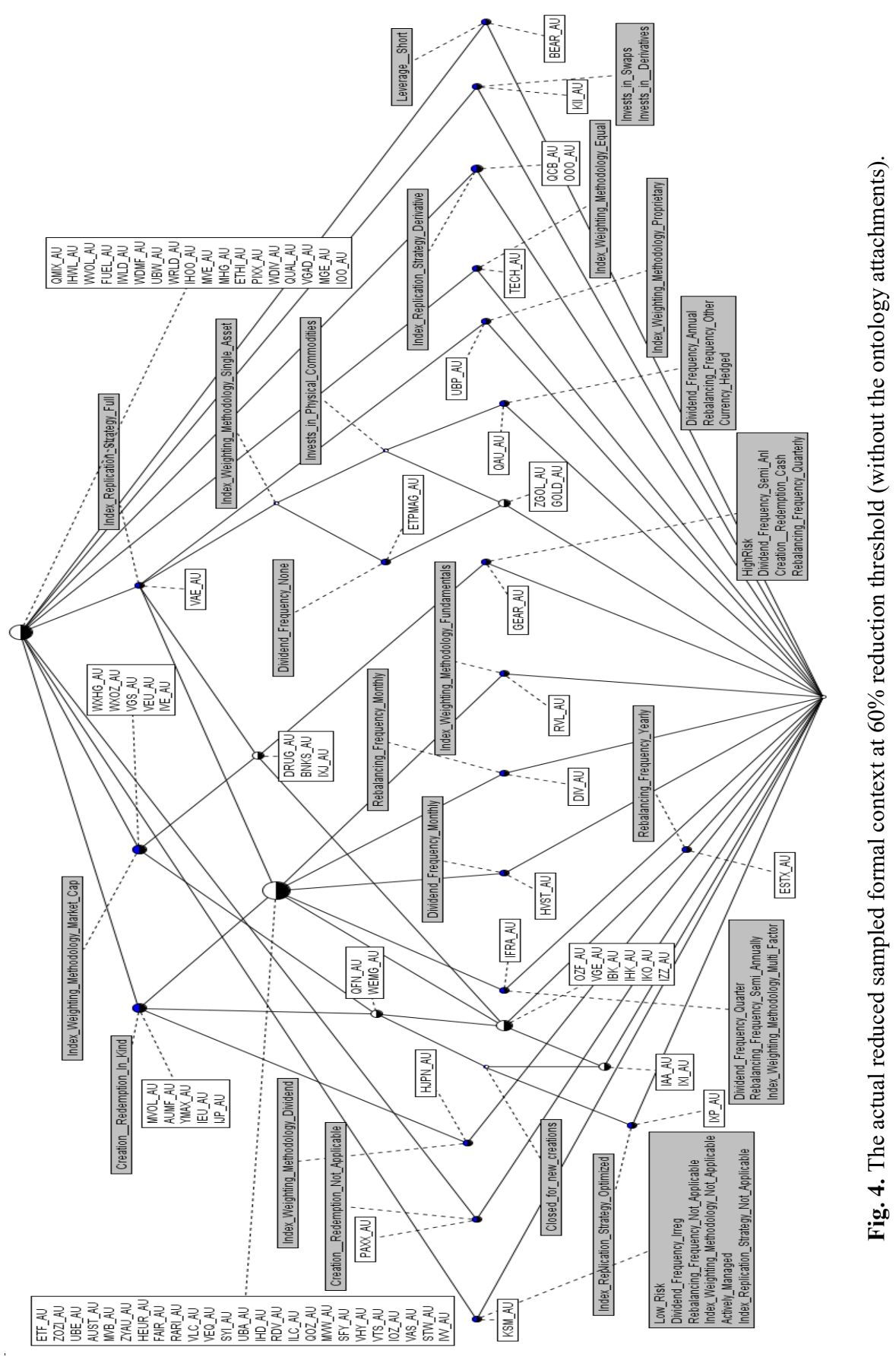




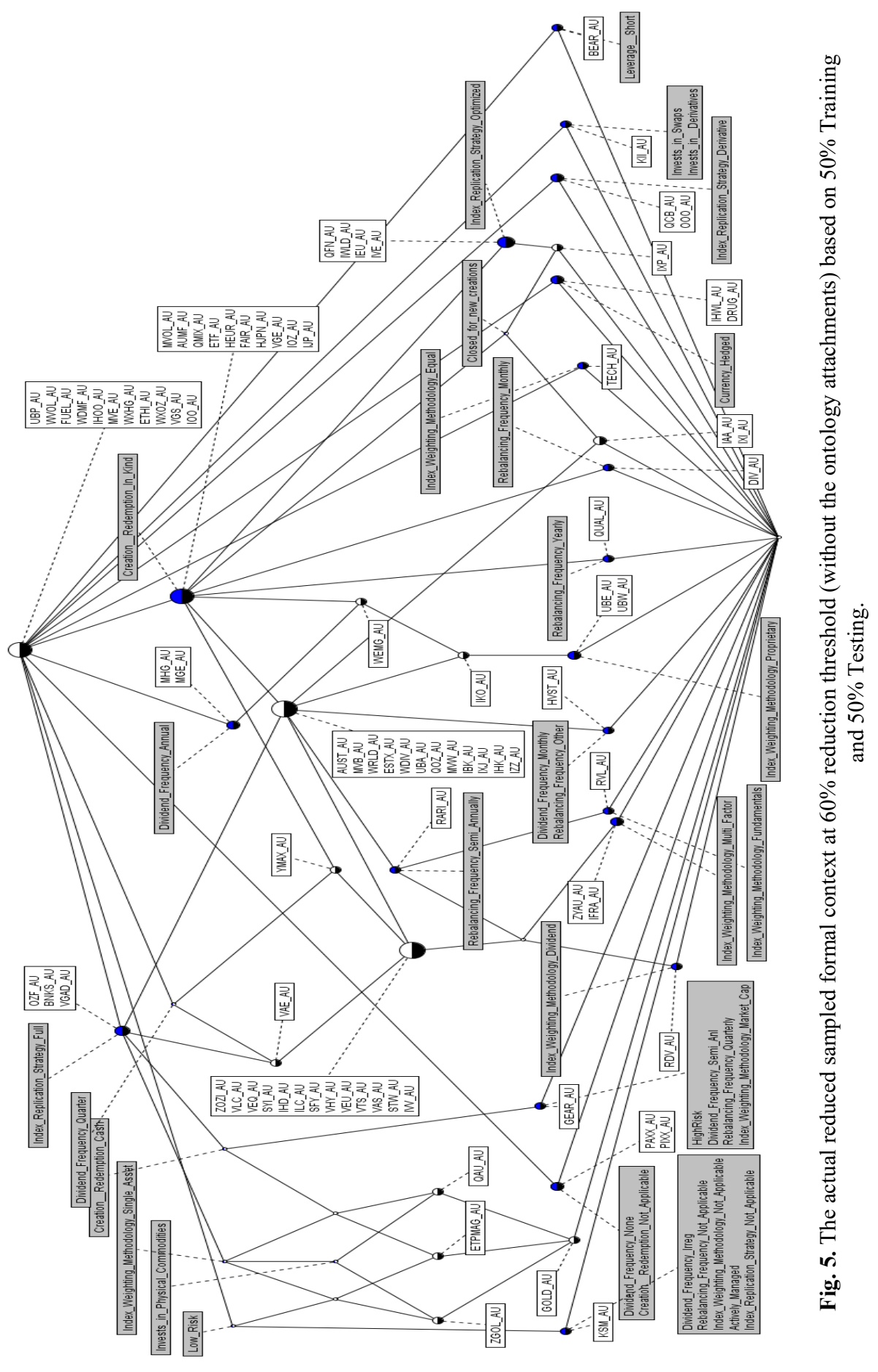




\section{Conclusion}

The financial institutions gather vast amount of data from various resources including financial market data and news data. However, to gain an advantage as a player in the market and obtain real benefits, the understanding of the meaning (semantics) of the data and the presentations of outcomes in simplified and relevant models are the key obstacles that need to be solved. Formal Concept Analysis (FCA) helps with the semantics by generating a lattice that comprises partial order relations between sets of properties (intent) and their instances (extent) in a domain that maps onto a semantic structure. The problem is the resultant lattice is too complex and noisy.

Using existing domain knowledge to inform and reduce a formal context (that is taken by FCA) is an opportunity that is being utilised in this work to simplify the resultant lattice and presents relevant models.

In this research, the Ontology-informed Lattice Reduction approach is applied to the financial domain as this approach relies on the use of an existing ontology to inform and reduce the financial sampled formal context based on different alignments and reductions measurements.

We specifically apply the approach to asset allocation problem in financial markets and assess the feasibility and validity of the different stages of the approach and the performance of the approach in regards to this real-world case study. The achieved results are good and pass all the testing measurements in producing creating a simplified, yet relevant, result that could be used in practice.

In the future work, we will (1) Extend the reduction approach to include the reasoning of ontology's description logics (DLs) to increase the accuracy of the reduction constrains. (2) Continue the work on the semantic attachments and add a "loopback" feature as a new extension that permits the utilisation of the approach outcomes to enrich the existing ontology.

\section{References}

1. De Mauro, A., Greco, M., Grimaldi, M.: A formal definition of Big Data based on its essential features. Library Review, 65(3), 122-135 (2016).

2. Singh, P. K., Kumar, C. A., Gani, A.: A comprehensive survey on formal concept analysis, its research trends and applications. International Journal of Applied Mathematics and Computer Science, 26(2), 495-516 (2016).

3. Rouane, M. H., Huchard, M., Napoli, A., Valtchev, P.: A proposal for combining formal concept analysis and description logics for mining relational data. In: International Conference on Formal Concept Analysis, pp. 51-65. Springer, Berlin, Heidelberg (2007).

4. Dias, S. M., Vieira, N. J.: Concept lattices reduction: Definition, analysis and classification. Expert Systems with Applications, 42(20), 7084-7097 (2015).

5. Ignatov, D. I.: Introduction to formal concept analysis and its applications in information retrieval and related fields. In: Braslavski, P., Karpov, N., Worring, M., Volkovich, Y., Ignatov, D. (eds.) Information Retrieval, vol. 505, pp. 42-141. Springer, Cham (2014).

6. Baader, F., Ganter, B., Sertkaya, B., Sattler, U.: Completing Description Logic Knowledge Bases Using Formal Concept Analysis. In: Proceedings of the 20th International Joint Conference on Artificial Intelligence (IJCAI), pp. 230-235. Hyderabad, India (2007). 
7. Stumme, G: Using ontologies and formal concept analysis for organizing business knowledge. In: Becker, J., Knackstedt, R. (eds.) Wissensmanagement mit Referenzmodellen, pp. 163-174. Physica, Heidelberg (2002).

8. Sarmah, A. K., Hazarika, S. M., Sinha, S. K.: Formal concept analysis: current trends and directions. Artificial Intelligence Review, 44(1), 47-86 (2015).

9. Quboa, Q., Behnaz, A., Mehandjiev, N., Rabhi, F., Petrounias I.:- Ontology-informed Lattice Reduction Using the Discrimination Power Index. Working paper under review. Dec 201

10. Behnaz, A., Natarajan, A., Rabhi, F. A., Peat, M.: A Semantic-Based Analytics Architecture and Its Application to Commodity Pricing. In International Workshop on Enterprise Applications and Services in the Finance Industry, pp. 17-31. Springer, Cham (2016).

11. LaValle, S., Lesser, E., Shockley, R., Hopkins, M. S., Kruschwitz, N.: Big data, analytics and the path from insights to value. MIT Sloan Management Review, 52(2), 21-32 (2011).

12. Gruber, T. R.: A translation approach to portable ontology specifications. Knowledge acquisition, 5(2), 199-220 (1993).

13. Financial Services Standards, http://www.omg.org/hot-topics/finance.htm, last accessed 2018/04/19.

14. Belohlavek, R., Trnecka, M.: Basic level of concepts in formal concept analysis. In: International Conference on Formal Concept Analysis, pp. 28-44. Springer, Berlin, Heidelberg (2012).

15. Singh, P. K., Kumar, C. A.: Concept lattice reduction using different subset of attributes as information granules. Granular computing, 2(3), 159-173 (2017).

16. Belohlavek, R., Vychodil, V.: Formal concept analysis with background knowledge: attribute priorities. IEEE Transactions on Systems, Man, and Cybernetics, Part C (Applications and Reviews), 39(4), 399-409 (2009).

17. Zhang, S., Guo, P., Zhang, J., Wang, X., Pedrycz, W.: A completeness analysis of frequent weighted concept lattices and their algebraic properties. Data \& Knowledge Engineering, 81, 104-117 (2012).

18. Bělohlávek, R., Sklenář, V., Zacpal, J.: Formal concept analysis with hierarchically ordered attributes. International Journal of General Systems, 33(4), 383-394 (2004).

19. Domenach, F., Portides, G.: Similarity Measures on Concept Lattices. In Analysis of Large and Complex Data, pp. 159-169. Springer, Cham (2016).

20. Choi, S. S., Cha, S. H., Tappert, C. C.: A survey of binary similarity and distance measures. Journal of Systemics, Cybernetics and Informatics, 8(1), 43-48 (2010).

21. W3C, SPARQL 1.1 Query Language, https://www.w3.org/TR/sparql11-query, last accessed 2018/04/23.

22. Sharpe, W. F.: Asset allocation: Management style and performance measurement. Journal of portfolio Management, 18(2), 7-19 (1992). 